\title{
Food allergies in children in the geographical area of Galati
}

\section{Camelia Bușilă, MD,PhD1,2, Gabriela Gurău, MD, PhD1,2, Mariana Stuparu-Crețu, MD, PhD1, Aurel Nechita, MD, PhD1,2, Maricica Gusa,2, Laura Candussi MD, PhD1,2}

\section{1"Dunarea de Jos" University of Galati, Faculty of Medicine and Pharmacy, Centre of Research in Medical-}

Pharmaceutical Field, Galati, Romania

2"Sf Apostol loan" Emergency Hospital for Children, Galati, Romania

\begin{abstract}
Background and aims: Food allergy is an abnormal response after a food ingestion, mediated by the immune system. Common food allergens are those from cow's milk proteins, eggs, peanuts, nuts, soybeans, wheat, fish, seafood. Food allergies affect $6 \%$ of children and $3-4 \%$ of adults, accounting for $29-50 \%$ of all cases of anaphylaxis, which produce 150-200 deaths annually. Cow's milk allergies affect 2$2.5 \%$ of children under 4 years of age.

Methods: The study group consisted of 414 patients admitted to "Sf Apostol loan" Emergency Hospital for Children, Galati during the period 2016-2017 for various illnesses, aged $1-18$ years, 228 boys (55\%) and 186 girls (45\%). Depending on the reason for hospitalization, patients sensitive to different allergens were classified in patients with respiratory diseases (63.98\%), digestive (16.42\%), dermatological (10.09\%), fever and various other diseases $(9.51 \%)$
\end{abstract}

\begin{tabular}{|c|c|c|}
\hline \multirow{2}{*}{$\begin{array}{c}\text { Subject } \\
\text { Category }\end{array}$} & \multicolumn{2}{|c|}{ Food Allergens } \\
\cline { 2 - 4 } & Level IgE, UI/ml/ & Range of variation, UI/ml \\
\hline \multicolumn{3}{|c|}{ Boys } \\
\hline $0-3$ & 2.77 & $0.36-64$ \\
\hline $3.0-6$ & 1.49 & $0.36-13.5$ \\
\hline $6.0-19$ & 1.73 & $0.36-56$ \\
\hline \multicolumn{3}{|c|}{ Girls } \\
\hline $0-3$ & 0.95 & $0.36-10.6$ \\
\hline $3.0-6$ & 1.67 & $0.36-12.5$ \\
\hline $6.0-19$ & 7.51 & $0.36-81$ \\
\hline
\end{tabular}

\section{Conclusions:}

- Most food allergens found in the studied group are cow's milk proteins, eggs, peanuts, nuts, soybeans, wheat, fish, seafood. Their detection is extremely important to prevent severe allergic reactions and anaphylactic shock.
Results: Gender distribution in groups of allergensensitized patients shows higher percentages in boys (47.10\%) relative to girls $(36.72 \%)$. The children we tested were especially sensitized to potato allergens (17.16\%), milk and milk proteins $(11.12 \%)$, peanuts $(5.00 \%)$, egg white $(4.24 \%)$, yolk $(3.58 \%)$. Sensitivity to food allergens has decreased with increasing age.

Most children tested had IgE specific antibody levels in cow's milk and main milk protein between $0.35 \mathrm{UI} / \mathrm{L}$ and $0.59 \mathrm{UI} / \mathrm{L}$ higher values have generally been found in children with more allergen sensitization. Egg sensitivity rate was low, high levels of $\lg E$ specific antibodies to egg components (egg white: 32 and $35 \mathrm{UI} / \mathrm{L}$ and yolk 13.5 and 5.6 UI / L) were found in poly-allergic patients ( 9 and 1 year, respectively).The peanut sensitization rate found by us was $5 \%$, being higher in boys (5.35\%) compared to girls $(4.43 \%)$, values close to those previously published.

A value of $15.5 \mathrm{UI} / \mathrm{L}$ found by us to a single child may indicate a high risk of permanent allergy to peanuts and possibly other family products (hazelnuts, nuts) by cross-reactivity. In our study, the rate of allergen sensitization in fish (mainly paralbumin and tropomyosin) between (0.36-1.1) $\mathrm{UI} /$ was not common in atopic children in Galati and was found in children over 3 years of age.

IgE specific concentrations of vegetables and fruits (potatoes, carrots, soybeans, wheat flour, rice, apple) were conditioned by the age of the child and they were generally low, with most values near the alert threshold $(0.35 \mathrm{UI} / \mathrm{L})$ and may indicate an allergy risk for sensitive patients to those plants.

- Analyzing comparatively the mean values of specific IgE in food allergens among the three age groups we found statistically significant differences only for $\beta$ lactoglobulin $(p=0.001)$ and egg white $(0.048)$. In the case of patients sensitized to food allergens between the two sexes, statistically significant differences were recorded for potato and casein $(p=0.049$, respectively 0.040$)$.

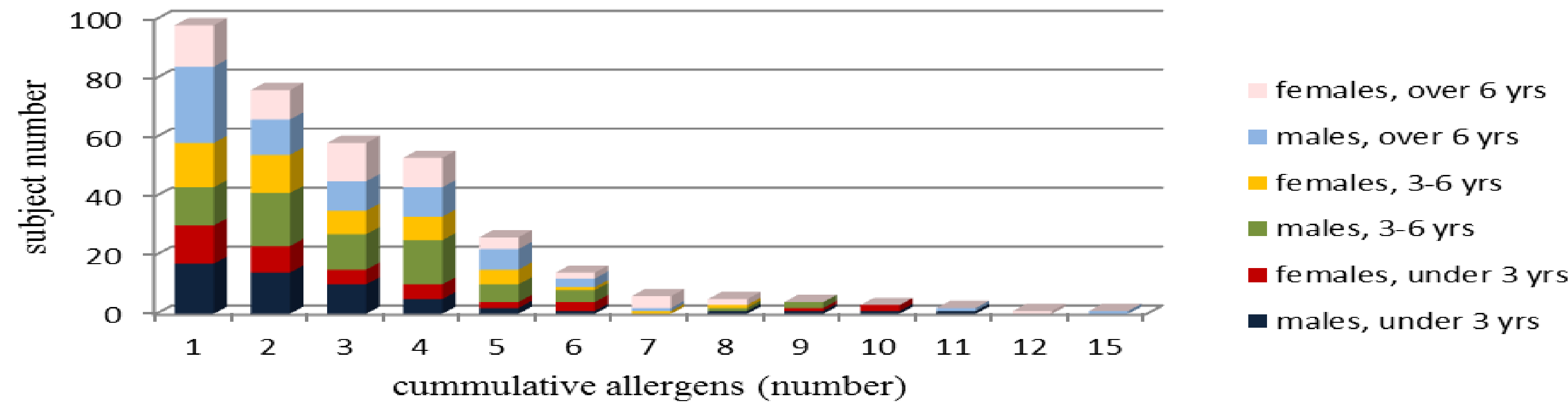

Variation in the prevalence of IgE positive tests with the age of investigated subjects

T1. hiGuandalini S., Newland C. Differentiating food allergies from food intolerances. Curr. Gastroenterol. Rep., 2011, 13(5):426-434. 